\title{
Лазер на основе германия с гибридной поверхностной плазменной модой
}

\author{
() А.А. Дубинов \\ Институт фризики микроструктур Российской академии наук, \\ 603950 Нижний Новгород, Россия \\ Нижегородский государственный университет им. Н.И. Лобачевского, \\ 603950 Нижний Новгород, Россия \\ E-mail: sanya@ipm.sci-nnov.ru
}

(Получена 27 апреля 2016 г. Принята к печати 10 мая 2016 г.)

Теоретически исследована возможность создания лазера на основе $n^{++}$-Ge с гибридной поверхностной плазменной ТМ-модой: вычислены распределение электромагнитных полей и коэффициент поглощения в рассматриваемой моде, фактор оптического ограничения, коэффициент усиления и пороговая плотность тока в рассматриваемом лазере. Показано, что при оптимальных толщинах слоев лазера на основе $n^{++}-\mathrm{Ge}$ c гибридной поверхностной плазменной ТМ-модой пороговая плотность тока может быть в 2-3 раза меньше, чем экспериментально наблюдаемая пороговая плотность тока в лазере на основе $n^{++}-\mathrm{Ge}$ с обычным диэлектрическим волноводом.

В настоящее время бурно развивается исследование лазеров с поверхностной плазменной ТМ-модой (surface plasmon) [1-4]. По аналогии с аббревиатурой LASER (Light Amplification by Stimulated Emission of Radiation) в литературе [1] стал употребляться и термин „спазер“ (SPASER - Surface Plasmon Amplification by Stimulated Emission of Radiation).

Интерес к „спазерам“ связан с возможностью генерации моды с сильной локализацией электрического поля по сравнению с длиной волны моды в вакууме, что может быть применимо в наноэлектронике. Поверхностные плазменные волны рассматриваются как очень перспективные носители информации, которые могут заменить электроны в интегральных схемах [5].

Кроме того, обычная поверхностная плазменная мода локализована около границы раздела двух сред, для которых выполняются следующие условия для величин диэлектрических проницаемостей: $\operatorname{Re}\left(\varepsilon_{1}\right)>0, \operatorname{Re}\left(\varepsilon_{2}\right)<0$, $\left|\operatorname{Re}\left(\varepsilon_{2}\right)\right|>\operatorname{Re}\left(\varepsilon_{1}\right)$ [6]. Необходимостью наличия только двух сред поверхностная плазменная мода отличается от мод обычного диэлектрического волновода, для которого необходимо наличие, как минимум, трех сред, причем реальная часть диэлектрической проницаемости центрального слоя должна превышать реальные части диэлектрической проницаемости ограничивающих сред. Это отличие можно использовать для создания лазера на основе $n^{++}$-Ge, выращенного на „родной“ Ge-подложке, что может привести к уменьшению плотности порогового тока из-за кардинального уменьшения дефектов в слое $n^{++}-\mathrm{Ge}$ по сравнению со слоем, выращенным на сильно рассогласованной по постоянной решетки кремниевой подложке.

Действительно, в существующих диодных лазе$\operatorname{pax}[7,8]$ с активной областью $n^{++}$-Ge ограничивающими слоями служат контактный слой $\mathrm{Si}$ и $\mathrm{Si}$-подложка, так как реальная часть диэлектрической проницаемости $\mathrm{Si}$ $\left(\varepsilon_{\mathrm{Si}}\right)$ значительно меньше, чем у Ge [9]. При этом из-за сильного рассогласования постоянных решеток $\mathrm{Si}$ и $\mathrm{Ge}$ слой $\mathrm{Ge}$ вырастает с большой плотностью дефектов (плотность дислокаций превышает $2 \cdot 10^{6} \mathrm{~cm}^{-2}[10]$ ). Это отрицательно сказывается на пороговой плотности тока, которая превышает 280 кА/ $\mathrm{cm}^{2}$ [7] в таком лазере, тогда как теоретическая оценка пороговой плотности тока (без учета влияния дефектов, связанных с $\mathrm{Si}$-подложкой) дает величину 5.8 кА/см ${ }^{2}$ [11]. Столь низкая оценка пороговой плотности тока связана также и с положительным влиянием рассогласования постоянных решеток $\mathrm{Si}$ и $\mathrm{Ge}$ : выращенный на $\mathrm{Si}$-подложке тонкий слой $\mathrm{Ge}$ растянут в плоскости, перпендикулярной оси роста структуры. Деформация растяжения приводит к уменьшению разрыва между нижележащей непрямой $L$-долиной и прямой Г-долиной зоны проводимости Ge с 136 до 100 мэВ [7], что увеличивает заселенность верхней прямой Г-долины (в которой и происходят оптические переходы между зоной проводимости и валентной зоной) и соответственно увеличивает коэффициент усиления при той же концентрации носителей в структуре [11]. Следует отметить, что для недеформированного Ge c концентрацией доноров $10^{20} \mathrm{~cm}^{-3}$, по оценке, пороговая плотность тока выше и составляет $21.1 \kappa \mathrm{A} / \mathrm{cm}^{2}$ [11], но все же меньше в 13 раз по сравнению с экспериментальным значением [7].

Однако использование обычной поверхностной плазменной моды (локализованной на границе, например, с алюминием) для лазера с активной областью $n^{++}-\mathrm{Ge}$ не представляется возможным. Можно показать, используя выражение для волнового вектора в такой моде из книги [6] $\left(\varepsilon_{\mathrm{Al}}\right.$ и $\varepsilon_{\mathrm{Ge}}-$ диэлектрические проницаемости алюминия и германия соответственно [9]):

$$
k=\frac{2 \pi}{\lambda} \sqrt{\frac{\varepsilon_{\mathrm{Al}} \varepsilon_{\mathrm{Ge}}}{\varepsilon_{\mathrm{Al}}+\varepsilon_{\mathrm{Ge}}}},
$$

что коэффициент поглощения излучения $(\alpha=2 \operatorname{Im}(k))$ на длине волны $\lambda=1.55$ мкм, которая соответствует прямому оптическому переходу в недеформированном $\mathrm{Ge}$, превышает $2450 \mathrm{~cm}^{-1}$ и значительно превышает возможный коэффициент усиления в таком лазере [11]. 
Ситуация меняется, если использовать не обычную, а гибридную поверхностную плазменную моду [12], которая существует для структур, содержащих тонкую прослойку (между металлом и полупроводником) среды с меньшей, чем у полупроводника, реальной частью диэлектрической проницаемости.

Рассмотрим структуру, состоящую из $p$-Ge подложки (обычно толщиной $\sim 300-500$ мкм), на которой выращен (в направлении $z$ ) активный слой $n^{++}$-Ge толщиной $d$ и покрытый последовательно слоем аморфного (не вносящего дефектов в слой $\mathrm{Ge}$ ) $n^{++}-\mathrm{Si}$ толщиной $d_{\mathrm{Si}}$ и алюминием.

Для расчета $z$-компоненты электрического поля $E_{z}$ и волнового вектора $k$ в ТМ-моде, распространяющейся в направлении $x$, можно записать уравнения Максвелла в следующем виде [13]:

$$
\begin{gathered}
\varepsilon(z) \frac{d}{d z}\left(\frac{1}{\varepsilon(z)} \frac{d H_{y}}{d z}\right)+\left[\varepsilon(z)\left(\frac{2 \pi}{\lambda}\right)^{2}-k^{2}\right] H_{y}=0, \\
E_{z}=-\frac{\lambda k}{2 \pi \varepsilon(z)} H_{y},
\end{gathered}
$$

где $\varepsilon(z)$ - пространственное распределение диэлектрической проницаемости в структуре:

$$
\varepsilon(z)= \begin{cases}\varepsilon_{\mathrm{Al}}, & z<0, \\ \varepsilon_{\mathrm{Si}}, & 0 \leq z<d_{\mathrm{Si}}, \\ \varepsilon_{\mathrm{Ge}}, & d_{\mathrm{Si}} \leq z<d_{\mathrm{Si}}+d, \\ \varepsilon_{\mathrm{Ge}}, & z \geq d_{\mathrm{Si}}+d .\end{cases}
$$

На границе слоев с разными диэлектрическими проницаемостями $H_{y}$ и $\frac{1}{\varepsilon(z)} \frac{d H_{y}}{d z}$ непрерывны. Также $H_{y} \rightarrow 0$ при $z \rightarrow \pm \infty$. Тогда уравнение для $k$ будет выглядеть следующим образом:

$$
\begin{aligned}
& \left(\varepsilon_{\mathrm{Si}} k_{\mathrm{Al}}-\varepsilon_{\mathrm{Al}} k_{\mathrm{Si}}\right)\left(\varepsilon_{\mathrm{Si}} k_{\mathrm{Ge}}-\varepsilon_{\mathrm{Ge}} k_{\mathrm{Si}}\right) \\
& \quad=\left(\varepsilon_{\mathrm{Si}} k_{\mathrm{Al}}+\varepsilon_{\mathrm{Al}} k_{\mathrm{Si}}\right)\left(\varepsilon_{\mathrm{Si}} k_{\mathrm{Ge}}+\varepsilon_{\mathrm{Ge}} k_{\mathrm{Si}}\right) \exp \left(2 k_{\mathrm{Si}} d_{\mathrm{Si}}\right),
\end{aligned}
$$

где $k_{\mathrm{Al}}=\sqrt{k^{2}-\varepsilon_{\mathrm{Al}}}, k_{\mathrm{Si}}=\sqrt{k^{2}-\varepsilon_{\mathrm{Si}}}$ и $k_{\mathrm{Ge}}=\sqrt{k^{2}-\varepsilon_{\mathrm{Ge}}}$.

На рис. 1 представлено вычисленное из выражений (2)-(5) пространственное распределение в структуре по координате $z$ реальной части квадратного корня диэлектрической проницаемости $(\operatorname{Re}(\sqrt{\varepsilon}))$ и квадрата модуля $E_{z}$ для двух случаев: $d_{\mathrm{Si}}=0$ (обычная поверхностная плазменная мода) и $d_{\mathrm{Si}}=31 \mathrm{Hм}$. На вставке к рис. 1 изображено распределение в структуре по координате $z$ квадрата модуля $E_{z}$ в большем масштабе. Из рисунка видно, что в случае $d_{\mathrm{Si}}=0$ компонента электрического поля $E_{z}$ сильно локализована на границе с металлом (на расстоянии от металла, меньшем 0.3 мкм). В то же время для случая $d_{\mathrm{Si}}=31 \mathrm{Hм}$ компонента электрического поля $E_{z}$ слабо локализована (на расстоянии от металла, меньшем 6 мкм). Значительно меньшая локализация плазменной моды во втором случае приводит к значительно меньшему ее поглощению в металле $\left(\alpha=110 \mathrm{~cm}^{-1}\right)$. Отметим, что из-за скачка в значениях диэлектрической проницаемости $\mathrm{Ge}$ и $\mathrm{Si}$, согласно (3), наблюдается скачок в величине $E_{z}$ в слое $\mathrm{Si}$.

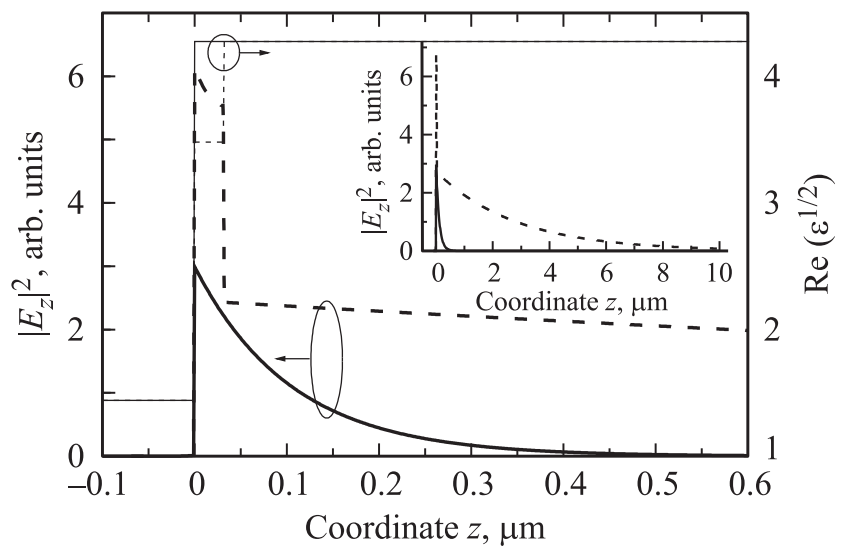

Рис. 1. Пространственное распределение $\operatorname{Re}(\sqrt{\varepsilon})$ и $\left|E_{z}\right|^{2}$ в структуре по координате $z$ для двух случаев: $d_{\mathrm{Si}}=0$ (сплошная кривая) и $d_{\mathrm{Si}}=31$ нм (штриховая кривая). На вставке распределение $\left|E_{z}\right|^{2}$ в структуре по координате $z$ в большем масштабе.

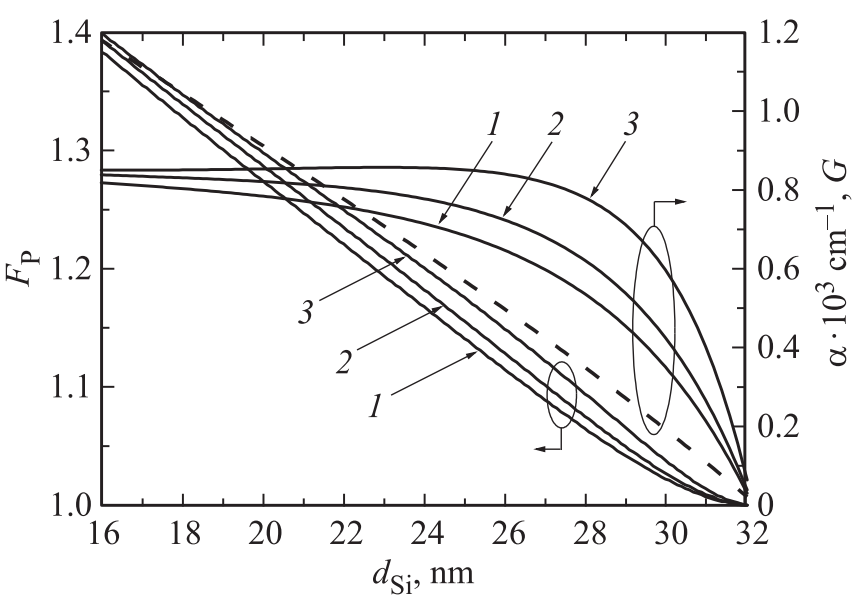

Рис. 2. Зависимость $\alpha$ (штриховая кривая) и $G, F_{\mathrm{P}}$ (сплошные кривые) от $d_{\mathrm{Si}}$, мкм: $1-0.7,2-0.9,3-1.5$.

Зависимость коэффициента поглощения $\alpha$ гибридной поверхностной плазменной моды от толщины кремниевой прослойки $d_{\mathrm{Si}}$ приведена на рис. 2 . Из рис. 2 видно, что $\alpha$ резко спадает с ростом $d_{\mathrm{Si}}$ и практически достигает нуля при стремлении $d_{\mathrm{Si}}$ к критическому значению (32.1 нм для данных параметров), при котором может существовать такая мода. Однако, для возможности генерации, критически важно то, что с ростом $d_{\mathrm{Si}}$ падает и величина фактора оптического ограничения (см. рис. 2), вычисляемого по формуле

$$
G=\frac{\int_{d_{\mathrm{si}}}^{d_{\mathrm{si}}+d}\left|E_{z}\right|^{2} d z}{\int_{-\infty}^{+\infty}\left|E_{z}\right|^{2} d z}
$$

и зависящего также от величины $d$. Следовательно, существуют оптимальные значения $d_{\mathrm{Si}}$ и $d$, при которых достигается минимальная пороговая плотность тока. 
Оценку для пороговой плотности тока, используя подход, развитый в работе [14], можно получить из следующего выражения:

$$
j_{\mathrm{th}}=q d\left(R_{A}+R_{d}+B_{L} F_{\mathrm{P}} n_{L} p+B_{\Gamma} F_{\mathrm{P}} n_{\Gamma} p\right),
$$

где $q-$ заряд электрона, $R_{A}-$ скорость оже-рекомбинации, $R_{d}-$ скорость безызлучательной рекомбинации на дефектах, связанных с легированием, $B_{L}$ и $B_{\Gamma}, n_{L}$ и $n_{\Gamma}-$ коэффициенты излучательной рекомбинации и концентрации электронов в $L$ - и Г-долинах соответственно, $p-$ концентрация дырок, $F_{\mathrm{P}}$ - фактор Парселла (см. рис. 2), определяемый для поверхностной плазменной моды следующим образом [3]:

$$
F_{\mathrm{P}}=1-\pi G \operatorname{Re}(k) \operatorname{Re}\left(k_{\mathrm{Ge}}\right) \lambda[d \operatorname{Re}(k) / d \lambda] / k_{s}^{3},
$$

где $k_{s}=2 \pi \operatorname{Re}\left(\sqrt{\varepsilon_{\mathrm{Ge}}}\right) / \lambda$.

Скорость оже-рекомбинации в объемных полупроводниках $R_{A}=C_{n} n^{2} p+C_{p} p^{2} n$, где $n=n_{L}+n_{\Gamma}$, а $C_{n}$ и $C_{p}-$ коэффициенты оже-рекомбинации, $R_{d}=(n+p) / \tau$, где $\tau$ - время безызлучательной рекомбинации на дефектах. Для расчетов использовались следующие значения параметров для германия, взятые из работы [11]: $B_{\Gamma}=1.3 \cdot 10^{-10} \mathrm{~cm}^{3} / \mathrm{c}, \quad B_{L}=5.1 \cdot 10^{-15} \mathrm{~cm}^{3} / \mathrm{c}$, $C_{n}=3 \cdot 10^{-32} \mathrm{~cm}^{6} / \mathrm{c}, C_{p}=7 \cdot 10^{-32} \mathrm{~cm}^{6} / \mathrm{c}, \tau=100 \mathrm{Hc}$.

Для нахождения пороговых значений концентраций электронов и дырок можно использовать выражение для порога генерации лазера [14]:

$$
g G=\alpha_{\text {in }} G+\alpha+\frac{1}{2 \Lambda} \ln \left(\frac{1}{R_{1} R_{2}}\right),
$$

где $g$ - коэффициент усиления активной среды, $\alpha_{\text {in }}$ коэффициент поглощения в активной среде, $\Lambda$ - длина лазера, $R_{1}$ и $R_{2}-$ коэффициенты отражения двух зеркал. В расчетах использовались следующие параметры лазера: длина волны излучения $\lambda=1.55$ мкм, $\Lambda=1 \mathrm{мм}, R_{1}=R_{2}=0.34$ (зеркало образовано разницей показателей преломления полупроводника и воздуха), температура полупроводника $T=300 \mathrm{~K}$, концентрация инжектированных электронов и дырок считалась равной. Коэффициент $g$ определяется следующей формулой $[11,14]$ :

$$
g=A \sqrt{2 \pi \hbar c / \lambda-E_{g}}\left[f_{n}-f_{p}\right] /(2 \pi \hbar c / \lambda),
$$

где $A=1.9 \cdot 10^{4}{ } \mathrm{~B}^{1 / 2} / \mathrm{cm}$,

$$
\begin{gathered}
f_{n}=\frac{1}{1+\exp \left\{\frac{\left(m_{R} / m_{n}\right)\left(2 \pi \hbar c / \lambda-E_{g}\right)-E_{\mathrm{F}}^{n}+E_{g}}{k_{\mathrm{B}} T}\right\}}, \\
f_{p}=\frac{1}{1+\exp \left\{-\frac{\left(m_{R} / m_{p}\right)\left(2 \pi \hbar c / \lambda-E_{g}\right)+E_{\mathrm{F}}^{p}}{k_{\mathrm{B}} T}\right\}},
\end{gathered}
$$

- функции распределения электронов и дырок, $c$ скорость света в вакууме, $\hbar$ и $k_{\mathrm{B}}$ - постоянные Планка

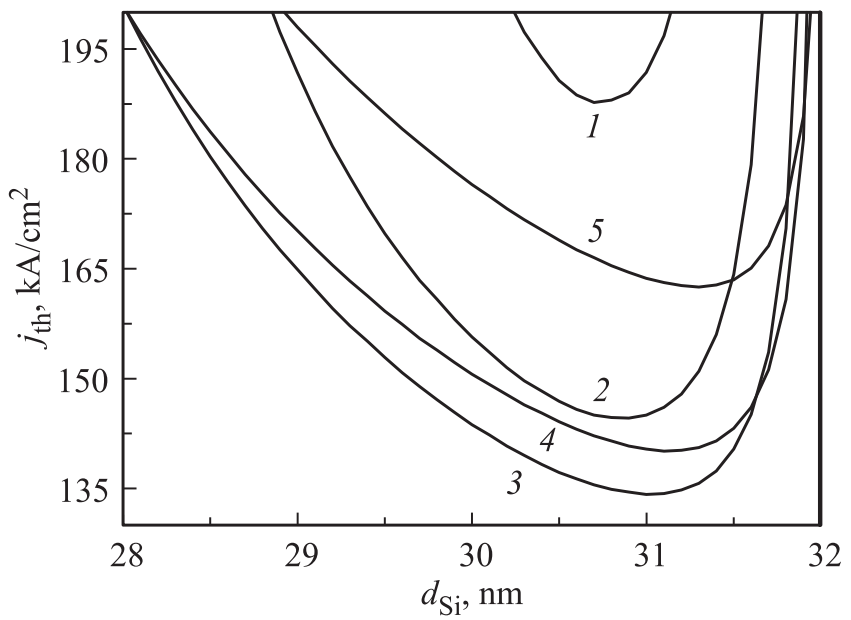

Рис. 3. Зависимость $j_{\text {th }}$ от $d_{\mathrm{Si}}$ для разных значений $d$, мкм: $1-0.6,2-0.7,3-0.9,4-1.1,5-1.5$.

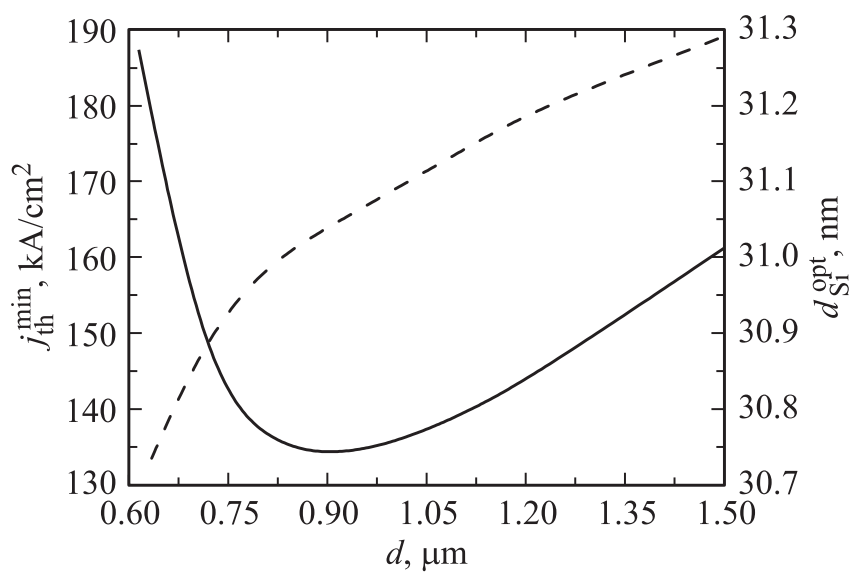

Рис. 4. Зависимость $j_{\mathrm{th}}^{\min }$ (сплошная кривая) и $d_{\mathrm{Si}}^{\mathrm{opt}}$ (штриховая кривая) от $d$.

и Больцмана соответственно, $m_{n}$ и $m_{p}-$ массы плотности состояний электронов в Г-долине и дырок соответственно, $m_{R}=m_{n} m_{p} /\left(m_{n}+m_{p}\right), R_{g}-$ ширина прямой запрещенной зоны полупроводника, отсчитываемая от потолка валентной зоны. Неравновесные квазиуровни Ферми для электронов $\left(E_{\mathrm{F}}^{n}\right)$ и дырок $\left(E_{\mathrm{F}}^{p}\right)$ в зависимости от концентраций донорной примеси $N$ и инжектируемых носителей $n_{\text {in }}$ можно найти из следующих выражений:

$$
\begin{aligned}
n= & \frac{2}{\hbar^{3}}\left(\frac{m_{n} k_{\mathrm{B}} T}{2 \pi}\right)^{3 / 2} \Phi_{1 / 2}\left(\frac{E_{\mathrm{F}}^{n}-E_{g}}{k_{\mathrm{B}} T}\right) \\
& +\frac{2}{\hbar^{3}}\left(\frac{m_{L} k_{\mathrm{B}} T}{2 \pi}\right)^{3 / 2} \Phi_{1 / 2}\left(\frac{E_{\mathrm{F}}^{n}-E_{g}^{L}}{k_{\mathrm{B}} T}\right)
\end{aligned}
$$

и

$$
p=\frac{2}{\hbar^{3}}\left(\frac{m_{p} k_{\mathrm{B}} T}{2 \pi}\right)^{3 / 2} \Phi_{1 / 2}\left(\frac{-E_{\mathrm{F}}^{p}}{k_{\mathrm{B}} T}\right)
$$

где $n=N+n_{\text {in }}, m_{L}-$ масса плотности состояний электронов в L-долине, $E_{g}^{L}-$ ширина непрямой запрещенной зоны полупроводника, $\Phi_{1 / 2}-$ интеграл 
Ферми-Дирака. Предполагается, что $N=10^{20} \mathrm{~cm}^{-3}$, что экспериментально достижимо $[15,16]$. Коэффициент $\alpha_{\text {in }}$ был найден эмпирически в работе [11]:

$$
\alpha_{\text {in }}=1.9 \cdot 10^{-18} n \lambda^{2.25}+6.2 \cdot 10^{-18} p \lambda^{2.43},
$$

где $\alpha_{\text {in }}$ измеряется в единицах $\mathrm{cm}^{-1}, n$ и $p$ измеряются в единицах $\mathrm{cm}^{-3}$, а $\lambda$ в единицах мкм.

Используя выражения (6), (8-13), можно найти величину пороговой плотности тока по формуле (7) в рассматриваемом лазере в зависимости от $d_{\mathrm{Si}}$ и $d$. На рис. 3 изображена зависимость $j_{\text {th }}$ от $d_{\mathrm{Si}}$ для нескольких значений $d$. Из рис. 3 видно, что для каждого значения $d$ существует минимальная пороговая плотность тока $j_{\text {th }}^{\min }$ при определенной оптимальной толщине $\mathrm{Si}$ прослойки $d_{\mathrm{Si}}^{\mathrm{opt}}$. Также наблюдается сильная чувствительность $j_{\text {th }}$ К величине $d_{\mathrm{Si}}$. При толщинах $d_{\mathrm{Si}}$, отличающихся от $d_{\mathrm{Si}}^{\mathrm{opt}}$ на $2 \mathrm{HM}, j_{\text {th }}$ увеличивается в 1.5 раза. Это еще лучше видно на рис. 4 , где представлена зависимость $j_{\mathrm{th}}^{\min }$ и $d_{\mathrm{Si}}^{\mathrm{opt}}$ от толщины активной области $d$. Из рис. 4 видно, что минимальное значение $j_{\text {th }}^{\min }=135 \mathrm{\kappa} / \mathrm{cm}^{2}$ достигается для $d=0.9$ мкм и $d_{\mathrm{Si}}^{\mathrm{opt}}=31 \mathrm{HM}$, а $d_{\mathrm{Si}}^{\mathrm{opt}}$ увеличивается с ростом $d$. Чувствительность $j_{\text {th }}$ к величине $d$ значительно слабее, чем к величине $d_{\mathrm{Si}}$.

В заключение можно сделать вывод, что в рассматриваемом лазере с гибридной поверхностной плазменной ТМ-модой минимальная пороговая плотность тока достигается при определенных оптимальных величинах толщины кремниевой прослойки $(31 \mathrm{Hм})$ и толщины активной области $n^{++}-\mathrm{Ge}(0.9$ мкм). Кроме того, значение пороговой плотности тока в рассматриваемом лазере может быть в 2-3 раза ниже по сравнению с экспериментальным значением пороговой плотности тока $n^{++}$-Ge лазера с диэлектрическим волноводом $[7,8]$.

Работа выполнена при поддержке РФФИ (№ 15-02-05272).

\section{Список литературы}

[1] M.A. Noginov, G. Zhu, A.M. Belgrave, R. Bakker, V.M. Shalaev, E.E. Narimanov, S. Stout, E. Herz, T. Suteewong, U. Wiesner. Nature, 460, 1110 (2009).

[2] R.-M. Ma, R.F. Oulton, V.J. Sorger, G. Bartal, X. Zhang. Nature Matetials, 10, 110 (2011).

[3] J.B. Khurgin, G. Sun. Appl. Phys. Lett., 100, 011105 (2012).

[4] D.Yu. Fedyanin, A.V. Arsenin. Opt. Express, 19, 12524 (2011).

[5] Е. Ozbay. Science, 311, 189 (2006).

[6] Л.Д. Ландау, Е.М. Лифшиц. Электродинамика сплошных сред (М., Наука, 1992)

[7] R.E. Camacho-Aguilera, Y. Cai, N. Patel, J.T. Bessette, M. Romagnoli, L.C. Kimerling, J. Michel. Opt. Express, 20, 11316 (2012).

[8] R. Koerner, M. Oehme, M. Gollhofer, M. Schmid, K. Kostecki, S. Bechler, D. Widmann, E. Kasper, J. Schulze. Opt. Express, 23, 14815 (2015).

[9] E.D. Palik. Handbook of optical constants of solids (N.Y., Academic Press, 1998).
[10] H.-C. Luan, D.R. Lim, K.K. Lee, K.M. Chen, J.G. Sandland, K. Wada, L.C. Kimerling. Appl. Phys. Lett., 75, 2909 (1999).

[11] J. Liu, X. Sun, D. Pan, X. Wang, L.C. Kimerling, T.L. Koch, J. Michel. Opt. Express, 15, 11272 (2007).

[12] R.F. Oulton, V.J. Sorger, D.A. Genov, D.F.P. Pile, X. Zhang. Nature Photonics, 2, 496 (2008).

[13] V.Ya. Aleshkin, A.A. Dubinov, V. Ryzhii. Письма ЖЭТФ, 89, 70 (2009).

[14] А.А. Дубинов. Письма ЖЭТФ, 97, 283 (2013).

[15] D.V. Yurasov, A.V. Antonov, M.N. Drozdov, V.B. Schmagin, K.E. Spirin, A.V. Novikov. J. Appl. Phys., 118, 145701 (2015).

[16] А.В. Антонов, М.Н. Дроздов, А.В. Новиков, Д.В. Юрасов. ФТП, 49, 1453 (2015).

Редактор А.Н. Смирнов

\section{A germanium laser with a hybrid surface plasmon mode}

\section{A.A. Dubinov}

Institute for Physics of Microstructures,

Russian Academy of Sciences,

603950 Nizhny Novgorod, Russia

Lobachevsky State University of Nizhny Novgorod, 603950 Nizhny Novgorod, Russia

Abstract A possibility to create $n^{++}$-Ge laser with hybrid surface plasmon TM-mode was studied theoretically. The distribution of electromagnetic fields and absorption coefficient in the given mode, the optical confinement factor, gain and threshold current density in this laser were calculated. It was shown, that for the optimum layer thickness of the $n^{++}$-Ge laser with hybrid surface plasmon TM-mode the threshold current density can be $2-3$ times lower than the experimentally observed threshold current density of $n^{++}$-Ge laser with usual dielectric waveguide. 